\title{
Experimental Characterization of Monolithic-Crystal Small Animal PET Detectors Read Out by APD Arrays
}

\author{
Marnix C. Maas, D. J. van der Laan, Dennis R. Schaart, Jan Huizenga, J. C. Brouwer, Peter Bruyndonckx, \\ Sophie Léonard, Cedric Lemaître, and Carel W. E. van Eijk, Member, IEEE
}

\begin{abstract}
Minimizing dead space is one way to increase the detection efficiency of small-animal PET scanners. By using monolithic scintillator crystals (e.g., $20 \mathrm{~mm} \times 10 \mathrm{~mm} \times 10 \mathrm{~mm}$ LSO), loss of efficiency due to inter-crystal reflective material is minimized. Readout of such crystals can be performed by means of one or more avalanche photo-diode (APD) arrays optically coupled to the crystal. The entry point of a gamma photon on the crystal surface can be estimated from the measured distribution of the scintillation light over the APD array(s). By estimating the entry point, correction for the depth-of-interaction (DOI) is automatically provided. We are studying the feasibility of such detector modules. To this end, a 64-channel test setup has been developed. Experiments to determine the effect on the spatial resolution of crystal surface finish and detector geometry have been carried out. The first results of these experiments are presented and compared to simulation results. The crystal surface finish has only a small influence on the spatial resolution. The spatial resolution of $20 \mathrm{~mm} \times 10$ $\mathrm{mm} \times 10 \mathrm{~mm}$ detectors is significantly better when read out on the front side than when read out on the back side. With a $20 \mathrm{~mm} \times 10$ $\mathbf{m m} \times 20 \mathbf{m m}$ crystal coupled to two APD arrays, a very small resolution degradation of only $\sim 0.2 \mathrm{~mm}$ is observed for an incidence angle of $30^{\circ}$ compared to normal incidence.
\end{abstract}

Index Terms-Angle of incidence, avalanche photodiode (APD), depth-of-interaction, monolithic scintillator crystals, nearest neighbor method, positron emission tomography (PET), spatial resolution.

\section{INTRODUCTION}

$\mathbf{R}$ ECENT research into small animal positron emission tomography (PET) detectors has put much emphasis on obtaining the best possible spatial resolution. The most important strategies for improving the spatial resolution have been to reduce the pixel size [1] and to include depth-of-interaction (DOI) information in the detector readout [2]. A second trend that can be observed is the development of multi-modality systems, combining functional imaging, such as PET, with anatomical imaging modalities, such as CT [3] or MR [4].

As the interest for dynamic and quantitative PET studies on small animals has increased, so has the need for PET systems

Manuscript received November 15, 2005; revised January 19, 2006. This research has been performed in collaboration with the Crystal Clear Collaboration, a CERN Technology Transfer Project grouping a number of European universities and government research institutes.

M. C. Maas, D. J. van der Laan, D. R. Schaart, J. Huizenga, J. C. Brouwer, and C. W. E. van Eijk are with the Delft University of Technology, 2629 JB Delft, The Netherlands (e-mail: m.c.maas@tnw.tudelft.nl).

P. Bruyndonckx, S. Léonard, and C. Lemaittre are with the Inter-University Institute for High Energies, Vrije Universiteit Brussel, 1050 Brussels, Belgium.

Digital Object Identifier 10.1109/TNS.2006.873711 with improved detection efficiency. The detection efficiency of current small animal PET systems is still limited, e.g., because of the use of arrays of small individual scintillation crystals separated by reflective material, resulting in a large fraction of dead space in the detector, or because the thickness of the crystal layer is not sufficient to absorb all gamma quanta. Furthermore, most systems employ photomultiplier tubes (PMTs), complicating dense packing of the modules and making it very difficult to use these scanners in strong magnetic fields, which is a severe limitation if the PET system is to be used in combination with an MR device.

As an alternative to detectors using crystal arrays, several types of detectors consisting of monolithic scintillation crystals read out by position sensitive photo-detectors have been proposed [5]-[8]. As these designs are generally difficult to implement in a scanner, or lack sufficient spatial resolution for small animal PET, we are currently investigating a new design based on monolithic scintillation crystals read out by one or more avalanche photo diode (APD) array(s) [9]-[11]. Readout of such detector modules is based on estimating the entry point of the annihilation quantum on the crystal's front surface from the light distribution measured by the APD array, using statistical methods or neural networks. Using monolithic crystals instead of arrays of individual crystals eliminates the need for inter-crystal reflective material, reducing dead space and increasing sensitivity. Furthermore, minimizing the dead space between modules may be easier with APDs than with photomultiplier tubes (PMTs). Also, because of their insensitivity to magnetic fields, the use of APDs makes it easier to combine the scanner with an MR device.

Possible drawbacks of this approach include the fact that the light from each scintillation event is shared among multiple APD pixels, reducing the average number of optical photons per APD pixel. The number of optical photons incident on a given APD pixel depends strongly on the location of the interaction point, causing a large dynamic range of the signals of interest. Furthermore, APDs provide a much lower gain than PMTs, necessitating the use of low-noise preamplifiers mounted close to the APDs.

We are studying the applicability in PET systems of detector modules based on the above readout principle, both by simulation and experiment. In this work, we present an experimental comparison between detectors with various geometries and crystal surface finishes. As an extension to previous exper- 


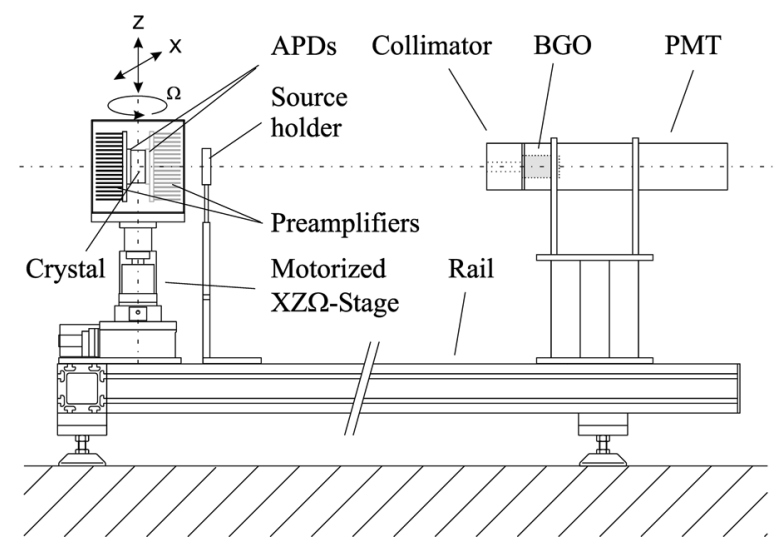

Fig. 1. Schematic representation of the experimental setup.

imental work [9], a new 64-channel setup has been built to accommodate more and different readout geometries, e.g., double sided readout. A statistical method for the estimation of the entry position of the annihilation quanta is used, instead of the neural networks used previously. Initially, experiments were carried out to optimize this position estimation method, increasing the sensitivity to physical parameters such as the geometry and the crystal surface roughness. Subsequently, experiments were performed to determine the influence of the detector geometry and the crystal surface finish on the spatial resolution. The results are compared to previous experimental work [9] and Monte Carlo simulations [10], [11].

\section{METHODS}

\section{A. Experimental Setup}

The experimental setup is depicted in Fig. 1. A scintillator crystal is coupled to one or two 32-channel $(8 \times 4)$ Hamamatsu S8550 APD array(s). Each APD array is mounted on a printed circuit board (PCB) holding 32 Cremat CR-110 preamplifiers. The crystal, APDs and PCBs are placed in a light tight, cubic Al box (outer dimensions $160 \mathrm{~mm} \times 160 \mathrm{~mm} \times 160 \mathrm{~mm}$ ), equipped with $0.5 \mathrm{~mm} \mathrm{Al}$ entrance windows.

A beam of $511 \mathrm{keV}$ annihilation photons emitted by a $\varnothing 0.5 \mathrm{~mm}^{22} \mathrm{Na}$ source irradiates the detector. The beam is defined by a second detector in coincidence with the APD detector, consisting of a $35 \mathrm{~mm}$ thick BGO crystal coupled to a PMT, with a $\varnothing 5.0 \mathrm{~mm}$ Pb-collimator ( $\mathrm{Pb}$ thickness 60 $\mathrm{mm})$. The PMT can be placed at a variable distance from the source. The detector can be scanned through the beam using a motorized $X Z \Omega$-stage, controlled by a PC.

The tail pulses from the preamplifiers are fed into four 16-channel spectroscopy amplifiers (CAEN N568B) through twisted pair flat cables. For each channel, these amplifiers have a fast output branch, consisting of a fixed gain single differentiation stage with a time constant of $100 \mathrm{~ns}$, and a slow branch, providing a semi-Gaussian pulse shape with an adjustable gain and a shaping time of $0.1 \mu \mathrm{s}, 0.2 \mu \mathrm{s}, 1.0 \mu \mathrm{s}$, or $3.0 \mu \mathrm{s}$. The signals from the slow outputs are transferred to two 32 channel, 12-bit, peak sensing ADCs (CAEN V785). Time pick-off on the APD signals is performed on the analog sum of the fast outputs of the spectroscopy amplifiers using a constant fraction discriminator (CFD, Ortec 934). The PMT anode signal is passed through an Ortec 474 timing filter amplifier, with both the differentiation and the integration switches set at Out (corresponding to time constants of $150 \mu \mathrm{s}$ and $10 \mathrm{~ns}$, respectively). Time pick-off on the resulting signal is again performed by a CFD (Ortec 934).

In all experiments, the APD arrays were kept at room temperature, and the shaping time of the slow branch of the amplifiers was set at $0.2 \mu$ s. The beam had an estimated diameter of $\sim 1.3 \mathrm{~mm}$ FWHM.

\section{B. Position Estimation}

When an annihilation photon interacts with the crystal, its entry point on the front surface of the crystal is estimated from the resulting distribution of scintillation light on the APD array(s), using a learning algorithm. Training data for the algorithm consists of light distributions acquired by irradiating the crystal with a beam of annihilation photons at many different positions with known coordinates.

Estimating the entry point of the annihilation photons on the front surface of the detector automatically provides a correction for the depth-of-interaction (DOI). An advantage of this approach is that it makes it possible to simply train the system using gamma beams with known entry points. It would be very difficult to train for the actual, three-dimensional position of interaction, since the depths of interaction of the individual photons in the training beam are unknown.

Consequences of this approach are that one must acquire training data for different angles of incidence, and that estimation of the entry point requires a-priori knowledge of the angle of incidence in order to be able to select the proper training set. In a scanner geometry, however, this angle can easily be estimated from the positions of the two detectors firing in coincidence. If necessary, this method can be refined by using the calculated entry points to derive an improved estimate of the angle of incidence, and repeating the position estimation using this improved estimate.

In the current experiments, after a crystal has been irradiated at a series of different positions, the training data is obtained by randomly splitting the measured data into two parts, a training set and a test set. Subsequently, events in the test set are assigned a coordinate by classifying them according to the nearest neighbor method [12]. That is, the least-squares difference of the light distribution of the event being estimated with all distributions in the training set is computed. The $N$ distributions in the training set that produce the smallest least-squares error are selected as the $N$ nearest neighbors, and the most frequently occurring coordinate within the nearest neighbor subset is assigned to the event being estimated. This procedure is repeated for all distributions in the test set.

An advantage of using a learning system to determine the entry points of the incident gamma quanta is that small gain non-uniformities between different APD pixels have little or no influence on the spatial resolution, because they are present in both the training set and the test set. As each APD array is different, each detector has to be individually trained. In principle, this is not very different from the crystal lookup table that needs 


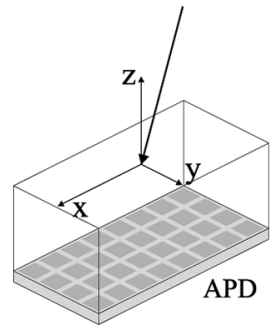

(a)

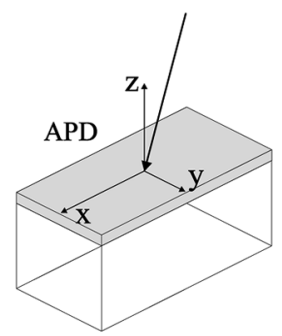

(b)

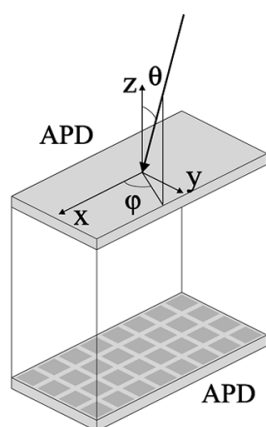

(c)
Fig. 2. Schematic representation of the detector geometries investigated: (a) back side, (b) front side, and (c) double readout. In (c), the coordinate system used to specify oblique angles of incidence is also indicated.

to be acquired for every array type detector in conventional PET systems.

Additionally, the nearest neighbor method has the advantage that for sufficiently large training sets, the probability of misclassification approaches the theoretical minimum: the Bayes error probability [12]. Therefore, the algorithm should yield results close to the best achievable with the given data, provided that enough training data and a suitable value of $N$ are used. This way, the influence of the algorithm on the results is minimized, maximizing the sensitivity to other parameters such as the readout geometry and the crystal surface finish.

\section{Estimation of Spatial Resolution}

In this work, the spatial resolution of the detectors is estimated using data from one-dimensional (1-D) scans. The beam is scanned along the long axis of the APD array (denoted as the $\mathrm{X}$-axis in the remainder of this work), perpendicular to its surface, through the center, from one edge of the crystal to the other in steps of $250 \mu \mathrm{m}$ (see Fig. 2). At each position, a fixed number of light distributions are recorded.

The data is then split into a training set and a test set, and the positions of the events in the test set are estimated using the algorithm described above. For each event in the test set, the positioning error, defined as the estimated minus the true coordinate, is calculated. To obtain an estimate for the spatial resolution, the positioning errors are collected in histograms. In the current experiments, these histograms are created five times, each time randomly splitting the data in a test set and a training set and estimating the entry positions of the test events. The means of the Full Width at Half Maximum (FWHM) and the Full Width at Tenth Maximum (FWTM) of these five histograms are used as estimates for the spatial resolution. As estimates of the uncertainty, the sample standard deviations of the mean FWHM and FWTM are used. Histograms of the position errors of all the test data are used for an overall figure of the spatial resolution. The position dependent spatial resolution can be estimated using histograms corresponding to individual beam positions.

\section{Optimization of the Position Estimation Algorithm}

To maximize the sensitivity of the spatial resolution to design parameters such as the detector geometry and the crystal surface finish, the influence of the position estimation algorithm should
TABLE I

OVERVIEW OF THE DIFFERENT SCINTILLATOR BLOCKS USED IN THE EXPERIMENTS

\begin{tabular}{llll}
\hline Block & Material & $\begin{array}{l}\text { Dimensions } \\
(\mathrm{mm} \times \mathrm{mm} \times \mathrm{mm})\end{array}$ & $\begin{array}{l}\text { Surface } \\
\text { Treatment }\end{array}$ \\
\hline I & LYSO:Ce $\mathrm{Ce}^{3+}$ & $20 \times 10 \times 10$ & polished \\
II & LSO:Ce ${ }^{3+}$ & $20 \times 10 \times 10$ & etched \\
III & LSO:Ce $\mathrm{Ce}^{3+}$ & $20 \times 10 \times 10$ & as-cut \\
IV & LYSO:Ce $\mathrm{Ce}^{3+}$ & $20 \times 10 \times 20$ & polished \\
V & LSO:Ce ${ }^{3+}$ & $20 \times 10 \times 20$ & etched \\
VI & LSO:Ce $\mathrm{Ce}^{3+}$ & $20 \times 10 \times 20$ & as-cut \\
\hline
\end{tabular}

be minimized, and, equally important, kept constant. The performance of the algorithm depends on the number of events per position used as training data $\left(n_{\operatorname{trn}}\right)$. Furthermore, for each $n_{\text {trn }}$, there is an optimum number of nearest neighbors $(\mathrm{N})$. An optimization of the algorithm was performed using a single data set, selecting a value for $n_{\text {trn }}$ and estimating the spatial resolution for multiple values of $\mathrm{N}$. This procedure was repeated for various values of $n_{\operatorname{trn}}$, until an optimum was reached.

\section{E. Comparison of Geometries and Surface Finishes}

A comparison of the performance of various detector geometries and crystal surface finishes was made using the scintillator crystals listed in Table I. The crystals marked as 'polished' were polished mechanically, the crystals marked as 'etched' were treated chemically according to a procedure described in [13]. The crystals marked as "as-cut" received no special surface treatment.

The blocks were investigated in various readout geometries. The 10-mm thick blocks (I, II, and III) were used either with an APD mounted on the front surface (i.e., the surface facing the gamma beam) or with an APD on the back surface (see Fig. 2). Experiments on the 20-mm blocks (IV, V, and VI) were performed with APDs on both the front and the back surfaces. All crystals were wrapped in Teflon on all sides, except where the APDs were coupled to the crystals. In all experiments, the number of events recorded per beam position was $3000, n_{\operatorname{trn}}$ was set at 1500 and $N$ was set at 200 .

\section{RESULTS AND DISCUSSION}

\section{A. Optimization of the Position Estimation Algorithm}

Fig. 3 shows the influence of the number of events per beam position used as training data $\left(n_{\text {trn }}\right)$ on the spatial resolution. For this graph, the resolution was calculated using the optimum $N$ corresponding to each particular value of $n_{\operatorname{trn}}$. This optimum was obtained using graphs like Fig. 4, showing the influence of $N$ on the resolution for a given value of $n_{\mathrm{trn}}$. Although for $n_{\operatorname{trn}}>1500$ a slight improvement may still be observed, considerations of both measurement and computation time for the position estimation lead to the choice of $n_{\operatorname{trn}}=1500$ and $N=200$ for subsequent experiments.

While during the optimization of the algorithm $n_{\text {tst }}$ was kept at 250 , in subsequent experiments $n_{\text {tst }}$ was set to 1500 , to improve the statistics in the histograms used for estimating the spatial resolution as a function of the beam position. Doing so has no significant influence on the overall spatial resolution, as is illustrated by the dashed curve in Fig. 3. This curve was obtained calculating the resolution at various values of $n_{\mathrm{tst}}$, using 


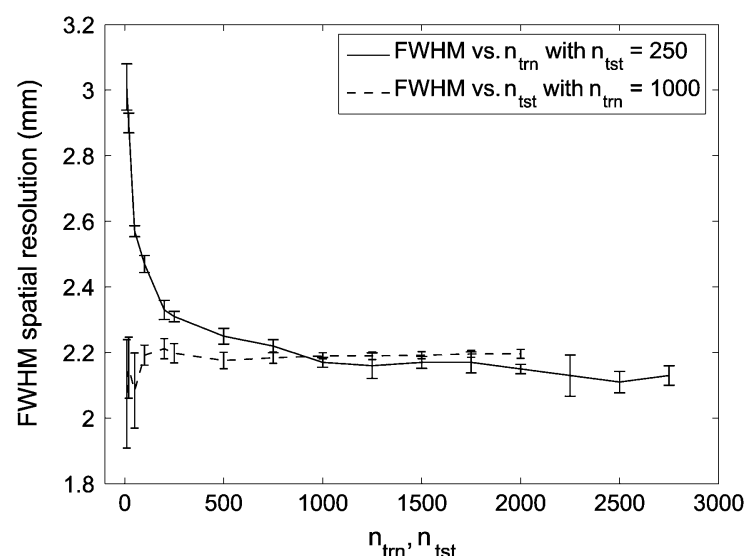

Fig. 3. Dependence of the spatial resolution on $n_{\mathrm{trn}}$ (solid line) with $n_{\mathrm{tst}}=$ 250 . At each $n_{\operatorname{trn}}$, the optimum $N$ for that particular $n_{\operatorname{trn}}$ was used. The dependence of the resolution on $n_{\mathrm{tst}}$, with $n_{\mathrm{trn}}=1000$ and $N=500$, is shown by the dashed line. These graphs were created using data from block I in the back-side readout geometry, containing 3000 events per beam position.

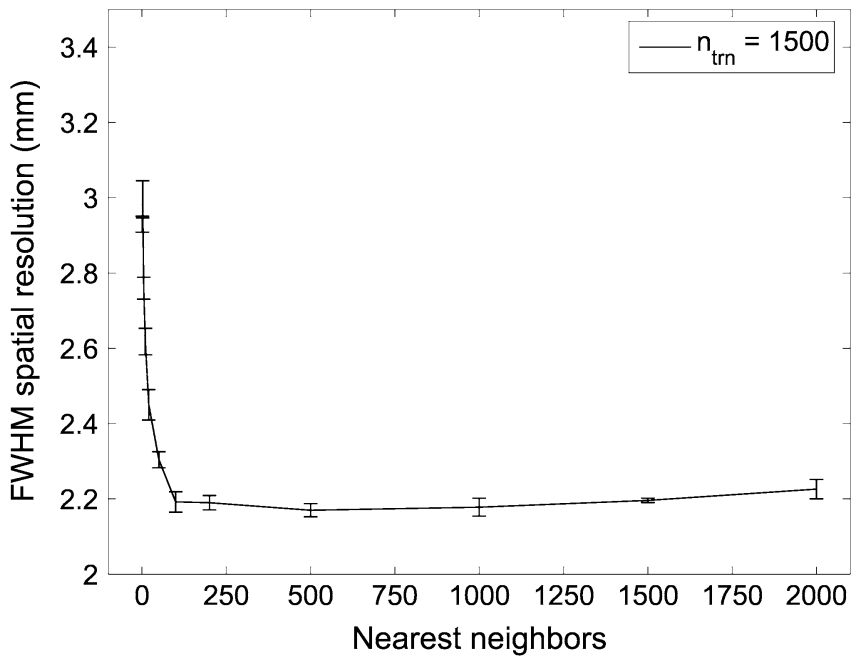

Fig. 4. Influence of the number of nearest neighbors $N$ on the spatial resolution for $n_{\mathrm{trn}}=1500$ and $n_{\mathrm{tst}}=250$. This graph was created using data from block $\mathrm{I}$ in the back-side readout geometry, containing 3000 events per beam position.

$n_{\text {trn }}=1000$ and $N=500$. Varying $n_{\text {tst }}$ may merely have an influence on the statistics in the error histograms, which can be is observed in Fig. 3, especially for $n_{\text {tst }}<200$. Above this value, the statistics in the overall error histogram are sufficient to accurately estimate the spatial resolution, and no influence on the resolution is observed.

\section{B. Comparison of Geometries and Surface Finishes}

The results of the comparison between various detector geometries and crystal surface finishes are summarized in Table II. Results from Monte-Carlo simulations [11], using a zero-width beam and neglecting electronic noise, are also listed for comparison. The experimental results, using $n_{\mathrm{trn}}=1500, n_{\mathrm{tst}}=$ 1500 , and $N=200$, show that polished and etched crystals perform slightly better than as-cut crystals, but that the influence of surface treatment is small. The simulation results do not show a significant influence of the surface treatment either.

The spatial resolution obtained with $10-\mathrm{mm}$ blocks when read out on the front side of the crystal [Fig. 2(b)] is consistently better than when read out on the back side [Fig. 2(a)], both in simulations and experiments. In $10 \mathrm{~mm}$ of LSO, approximately $61 \%$ of the $511 \mathrm{keV}$ photons that interact with the crystal do so within the first $5 \mathrm{~mm}$, due to the exponential decrease of the interaction probability as a function of depth. Simulation results presented elsewhere [11] show that the spatial resolution improves when events take place at smaller distances from the APD array, which may explain these results. The improved light collection in the front-side readout geometry apparently outweighs the increased scattering due to the presence of the APD array and the PCB materials in the beam. This is not surprising, since the APD array essentially consists of a piece of $\mathrm{Si}$ of the order of $\sim 100 \mu \mathrm{m}$ thick mounted on a $\sim 1 \mathrm{~mm}$ alumina substrate, resulting in a small interaction probability for $511 \mathrm{keV}$ photons.

The experiments with 20 -mm thick crystals read out on both sides yield resolutions only slightly worse than with $10-\mathrm{mm}$ crystals read out on the front side, but better than with $10-\mathrm{mm}$ crystals read out on the back side. The simulation results show the same trend [11]. Although the 10-mm LSO crystals read out on the front side perform slightly better, it is noted that the probability for a $511 \mathrm{keV}$ photon perpendicularly incident on the crystal to undergo any interaction (photoelectric or Compton) is only $\sim 58 \%$. Increasing the crystal thickness to $20 \mathrm{~mm}$ increases this probability to $\sim 83 \%$ [14]. It is noted that because of the large dimensions of the crystals, a large fraction of the detected events is observed in the full-energy peak, due to photons that are re-absorbed in the crystal after undergoing (multiple) Compton-scattering. Both in experiments and Monte Carlo simulations, $\sim 70 \%$ of the detected events are observed in the fullenergy peak for 10-mm LSO crystals, although the photo-fraction of $511 \mathrm{keV}$ photons in LSO is $\sim 30 \%$. For 20 -mm crystals, $\sim 75 \%$ of the detected events are observed in the full-energy peak. Thus, increasing the crystal thickness improves the detection efficiency considerably, a significant advantage for high efficiency PET systems.

Table II also shows results for 20-mm thick blocks read out on the front surface only. These results have been obtained from the same data set as the double-readout results, but neglecting the information of the backside APD. Compared to double-sided readout, the resolution is degraded only by about $0.06 \mathrm{~mm}$ to $0.14 \mathrm{~mm}$. Readout on the back surface only, on the other hand, degrades the spatial resolution much more, as is exemplified by the FWHM of $4.14 \mathrm{~mm}$ obtained for block IV. This is consistent with the observation that front-side readout on $10-\mathrm{mm}$ crystals performs better than back-side readout.

Fig. 5 shows the spatial resolution as a function of the beam position for several geometries: block I read out on the back side, block I read out on the front side and block IV read out on both sides. In the same figure, the result of a simulation on a 20-mm thick polished, Teflon-clad LSO crystal read out on both sides, assuming a zero-width beam and neglecting electronic noise, is presented. Fig. 6 shows a similar graph, comparing the different surface finishes: the lines represent blocks I, II, and III, each read out on the front side. All the experimental graphs show a degradation of the spatial resolution towards the edge of the detector. This effect is also observed in the simulation results.

Previous results showed that increasing the angle of incidence from $0^{\circ}$ to $40^{\circ}$ on a monolithic-crystal detector similar to block 
TABLE II

Comparison of Geometries and Surface Finishes. The Simulated Results Were Obtained Assuming a Zero-Width Beam and Neglecting Electronic Noise [11]. The Uncertainties Stated Are the Standard Deviation on the FWHM Spatial Resolution Obtained From DifFerent Reconstructions Performed on the Same Data SeT

\begin{tabular}{|c|c|c|c|c|c|}
\hline \multirow[t]{2}{*}{ Block } & \multirow{2}{*}{$\begin{array}{l}\text { Readout } \\
\text { geometry }\end{array}$} & \multicolumn{2}{|c|}{ Experiment } & \multicolumn{2}{|c|}{ Simulation } \\
\hline & & fwhm (mm) & fwtm (mm) & fwhm (mm) & fwtm (mm) \\
\hline I & front & $1.96 \pm 0.03$ & $4.78 \pm 0.04$ & $0.572 \pm 0.007$ & $2.52 \pm 0.01$ \\
\hline I & back & $2.19 \pm 0.02$ & $5.21 \pm 0.03$ & $0.858 \pm 0.004$ & $3.29 \pm 0.02$ \\
\hline II & front & $2.01 \pm 0.01$ & $4.92 \pm 0.04$ & & \\
\hline II & back & $2.17 \pm 0.03$ & $5.24 \pm 0.04$ & & \\
\hline III & front & $2.05 \pm 0.02$ & $5.21 \pm 0.06$ & & \\
\hline III & back & $2.35 \pm 0.01$ & $5.99 \pm 0.04$ & $0.856 \pm 0.009$ & $3.42 \pm 0.02$ \\
\hline IV & double & $2.06 \pm 0.02$ & $5.44 \pm 0.05$ & $0.692 \pm 0.004$ & $2.91 \pm 0.02$ \\
\hline IV & front only & $2.20 \pm 0.03$ & $7.30 \pm 0.05$ & & \\
\hline IV & back only & $4.14 \pm 0.10$ & $12.41 \pm 0.15$ & & \\
\hline V & double & $2.06 \pm 0.01$ & $5.44 \pm 0.06$ & & \\
\hline V & front only & $2.16 \pm 0.06$ & $7.24 \pm 0.07$ & & \\
\hline VI & double & $2.10 \pm 0.02$ & $5.62 \pm 0.03$ & $0.626 \pm 0.007$ & $2.88 \pm 0.02$ \\
\hline VI & front only & $2.16 \pm 0.04$ & $7.46 \pm 0.07$ & & \\
\hline
\end{tabular}

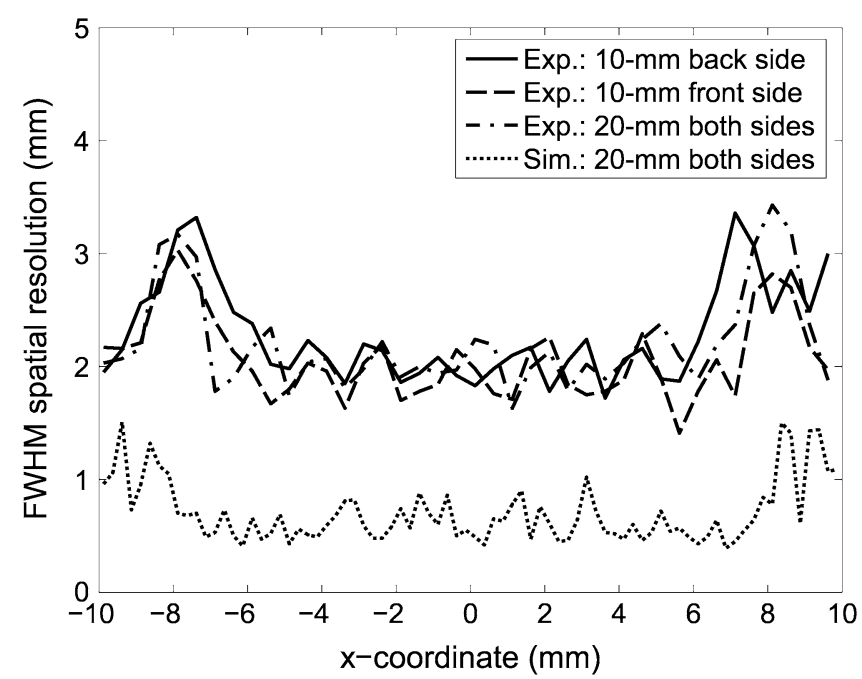

Fig. 5. Comparison of the measured spatial resolution as a function of the beam position for several detector geometries: a 10-mm thick polished LYSO block read out on the front, or on the back surface, and a 20-mm thick polished LYSO block read out on both sides. For comparison, a curve representing a simulation of a $20-\mathrm{mm}$ thick polished LSO block read out on both sides, assuming a zerowidth beam and neglecting electronic noise, is also included. The edges of the crystal are located at -10 and $10 \mathrm{~mm}$. The resolution at each beam position was determined from the combined histogram of 5 reconstructions on the same data set. In the experimental curves, the data has been rebinned into sections of $0.5 \mathrm{~mm}$ each.

I in the back-side readout geometry caused a deterioration of the spatial resolution of only $\sim 0.6 \mathrm{~mm}$ FWHM, compared to a degradation of more than $5 \mathrm{~mm}$ FWHM when a detector based on an array of individual crystals was used [9]. Fig. 7 shows the angular dependence of the spatial resolution of block IV in the double-readout geometry. From $0^{\circ}$ to $30^{\circ}$, the spatial resolution deteriorates by less than $0.2 \mathrm{~mm}$ FWHM. Comparison of these results to [15] suggests that the 20-mm double readout geometry may be slightly less affected by an increased angle than the 10 -mm back side geometry. In the latter case, the average interaction distance to the APD increases with increasing angle, causing more deterioration of the resolution than when an APD would be present on the front surface also.

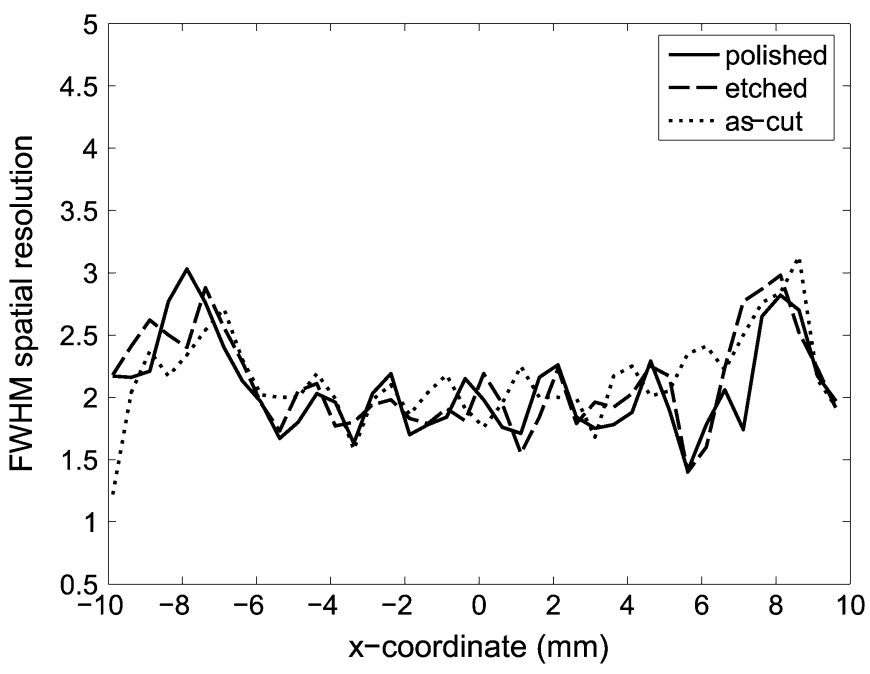

Fig. 6. Spatial resolution as a function of the beam position of three $20 \mathrm{~mm} \times 10 \mathrm{~mm} \times 10 \mathrm{~mm}$ scintillator blocks with an APD mounted on the front surface: polished LYSO (solid line), etched LSO (dashed line) and as-cut LSO (dotted line). In these curves, the data has been rebinned into sections of $0.5 \mathrm{~mm}$ each.

\section{CONCLUSIONS AND OUTLOOK}

Optimization of the position estimation algorithm and its parameters showed that the method of position estimation may have a strong influence on the spatial resolution obtained. The method presently used was chosen because it was expected to yield results close to the optimum, although the development of even better algorithms of course remains possible. The results obtained with this algorithm are promising and compare well to previously obtained results using neural networks [15]. The method's consistent performance and relatively simple optimization procedure facilitated the research of detector design parameters while minimizing the influence of the algorithm on the results. It is to be noted, however, that the nearest neighbor method may have limited applicability in practical PET systems, as the position estimation procedure is computationally intensive. For practical use in a PET scanner, neural networks have the advantage that the position estimation occurs much faster. 


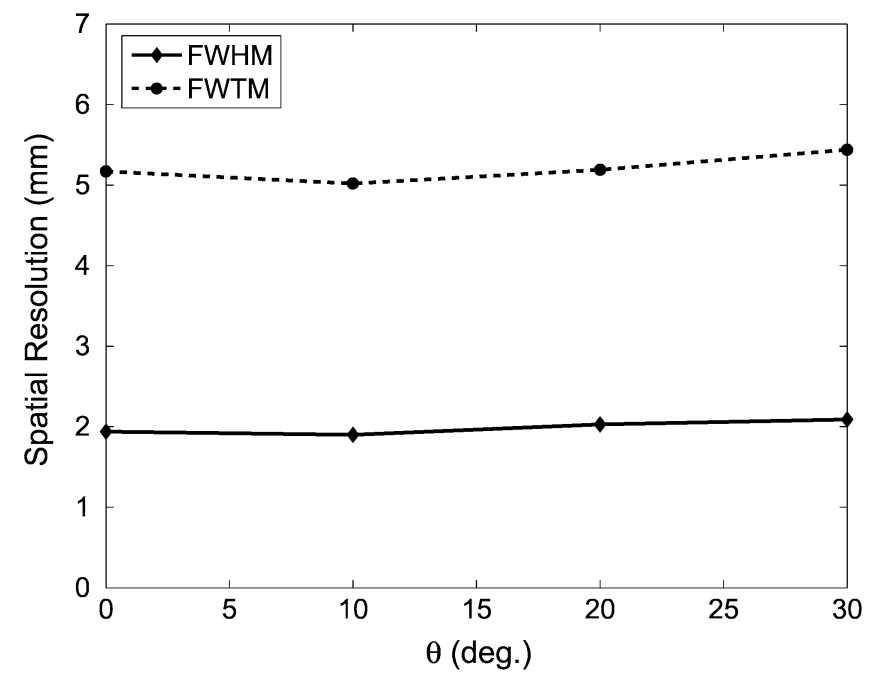

Fig. 7. Spatial resolution as a function of $\theta$ of a $20 \mathrm{~mm} \times 10 \mathrm{~mm} \times 20 \mathrm{~mm}$ polished, Teflon wrapped LYSO crystal (block IV) read out on both sides with $\phi=0^{\circ}$. It is noted that the resolution at $\theta=0^{\circ}$ is slightly different than listed in Table II. This graph concerns a different measurement performed with a slightly smaller beam diameter, which may account for the difference.

The similarity of the results obtained with both methods is therefore very convenient.

A comparison of various surface treatments of the crystals showed little influence on the spatial resolution. For future PET systems this could be an advantage, as the cost for crystal manufacturing may be reduced by avoiding relatively expensive surface treatments such as mechanical polishing.

The read-out geometry, on the other hand, exhibits a significant influence on the spatial resolution. For $10-\mathrm{mm}$ thick crystals, front-side readout consequently yields a better resolution than back-side readout, although in a practical PET scanner the implementation of front-side readout makes the design somewhat more complicated. Furthermore, the detection efficiency of $10-\mathrm{mm}$ thick crystals is considerably lower than that of $20-\mathrm{mm}$ thick crystals.

Therefore, it is very favorable that the 20 -mm thick crystals with double-sided read-out also show good results, not only at normal incidence, but also at angles of incidence up to $30^{\circ}$. The small influence of the angle of incidence on the spatial resolution implies that the intrinsic correction for the DOI provided by the method used to estimate the photon entry points is very effective. This is of great importance for imaging larger objects, as it reduces the parallax error, thus allowing off-axis points to be imaged without loss of spatial resolution.

It is to be noted, however, that estimation of the photon entry points requires a-priori knowledge of the angle of incidence to select the proper training set for position estimation. In a scanner geometry, this angle can in principle be estimated from the positions of the detectors triggering in coincidence. However, in a typical small-animal PET geometry with a ring diameter of $\sim 12 \mathrm{~cm}$ and detectors with an axial or radial extent of $20 \mathrm{~mm}$, an angular uncertainty of up to $\sim 10^{\circ}$ may exist between any detector pair. Therefore, it may be necessary to repeat the estimation of the entry points, using an improved estimate of the angle of incidence using the entry points obtained from an initial estimate of the angle. The accuracy of the angle of incidence needed for an accurate estimation of the photon entry points will be the subject of future investigations.

The simulation results presented in this work yield considerably better spatial resolutions than the experiments, due to the absence of electronic noise in the simulations and the assumption of a zero-width beam. These idealizations were applied to maximize the influence of variations in crystal surface and readout geometry in the simulations. Despite the difference in absolute values, the trends concerning crystal surface and readout geometry observed in simulations and experiments are similar.

The simulation results suggest that the finite diameter of the test beam significantly adds to the observed FWHM spatial resolution. This is confirmed by the observation that measurements with slightly different beam widths yield different spatial resolutions, see e.g., the caption of Fig. 7. We are currently working to quantify the influence of the beam diameter on the measured spatial resolution, and we aim to report on the intrinsic detector resolution (i.e., corrected for the beam width) in future work.

In the present paper, we have focused on the influence of several design parameters of monolithic scintillator detectors on the spatial resolution. In the near future, the energy resolution and timing resolution will also be reported on. In addition, the angular dependence of the spatial resolution will be studied in more detail. Furthermore, the detection efficiency of monolithic scintillator detectors will be compared directly to that of more traditional detectors with the same overall size, consisting of arrays of individual crystals. Although monolithic scintillator detectors offer a clear advantage in terms of dead space, in principle it might be that this advantage is partly lost due to the reduced signal to noise ratio resulting from the sharing of scintillation light among multiple APD pixels. Finally, we aim to extend our research of detector performance to other scintillator materials, such as $\mathrm{LaBr}_{3}$. Because of its high light yield and fast response, this material could be of interest for PET applications.

\section{ACKNOWLEDGMENT}

The authors would like to thank C. Woody of Brookhaven National Laboratory for kindly supplying the printed circuit boards used for reading out the APD arrays.

\section{REFERENCES}

[1] Y. C. Tai, "MicroPET II: Design, development, and initial performance of an improved microPET scanner for small-animal imaging," Phys. Med. Biol., vol. 48, pp. 1519-1537, May 2003.

[2] U. Heinrichs, U. Pietrzyk, and K. Ziemons, "Design optimization of the PMT-ClearPET prototypes based on simulation studies with GEANT3," IEEE Trans. Nucl. Sci., vol. 50, no. 5, pp. 1428-1432, Oct. 2003.

[3] M. Khodaverdi, "Preliminary studies of a micro-CT for a combined small animal PET/CT scanner," in Proc. IEEE NSS, 2001, vol. 3, pp. 1605-1606.

[4] R. B. Slates, S. R. Cherry, A. Boutefnouchet, Y. Shao, M. Dahlbom, and K. Farahani, "Design of a small animal MR compatible PET scanner," IEEE Trans. Nucl. Sci., vol. 46, no. 3, pp. 565-570, Jun. 1999.

[5] S. Delorme, R. Frei, C. Joseph, J.-F. Loude, and C. Morel, "Use of a neural network to exploit light division in a triangular scintillation crystal," Nucl. Instrum. Meth. A, vol. 373, pp. 111-118, 1996.

[6] D. Clément, R. Frei, J.-F. Loude, and C. Morel, "Development of a 3D position sensitive scintillation detector using neural networks," in Proc. IEEE NSS, 1998, vol. 3, pp. 1448-1452. 
[7] L. E. Adam, J. S. Karp, and M. E. Daube-Witherspoon, "Evaluation of performance of the CPET scanner using standardized measurement techniques," in Proc. IEEE NSS, 2000, vol. 3, pp. 17/46-17/50.

[8] P. P. Antich, N. Malakhov, R. Parkey, N. Slavin, and E. Tsyganov, "3D position readout from thick scintillators," Nucl. Instrum. Meth. A, vol. 480, pp. 782-787, 2002.

[9] P. Bruyndonckx, S. Léonard, J. Liu, S. Tavernier, P. Szupryczynski, and A. Fedorov, "Study of spatial resolution and depth of interaction of APD-based PET detector modules using light sharing schemes," IEEE Trans. Nucl. Sci., vol. 50, no. 5, pp. 1415-1419, Oct. 2003.

[10] D. J. van der Laan, M. C. Maas, D. R. Schaart, P. Bruyndonckx, S. Léonard, and C. W. E. van Eijk, "Performance optimization of continuous scintillator PET detector modules using Cramér-Rao theory combined with Monte Carlo simulations," in Proc. IEEE NSS MIC, 2004, vol. 4, pp. 2417-2421.
[11] — - "Using Cramér-Rao theory combined with Monte Carlo simulations for the optimization of monolithic scintillator PET detectors," IEEE Trans. Nucl. Sci., to be published.

[12] T. M. Cover and P. E. Hart, "Nearest neighbor pattern classification," IEEE Trans. Inf. Theory, vol. IT-13, no. 1, pp. 21-27, Jan. 1967.

[13] R. Slates, A. Chatziioannou, B. Fehlberg, T. Lee, and S. Cherry, "Chemical polishing of LSO crystals to increase light output," IEEE Trans. Nucl. Sci., vol. 47, no. 3, pp. 1018-1023, Jun. 2000.

[14] C. W. E. van Eijk, "Inorganic scintillators in medical imaging," Phys. Med. Biol., vol. 47, pp. R85-R106, 2002.

[15] P. Bruyndonckx, S. Léonard, S. Tavernier, C. Lemaître, O. Devroede, Y. Wu, and M. Krieguer, "Neural network-based position estimators for PET detectors using monolithic LSO blocks," IEEE Trans. Nucl. Sci., vol. 51, no. 5, pp. 2520-2525, Oct. 2004. 